\title{
Interfaces of Xe Inclusions Ion-implanted in Al --- Ordering in a Fluid Xe and Matrix Oxidation at Surfaces
}

\author{
Kazuo Furuya ${ }^{*}$, Konstantin Iakoubovskii*, Kazutaka Mitsuishi*, Stephen E. Donnelly ${ }^{* *}$ and Minghui Song ${ }^{*}$ \\ ${ }^{*}$ National Institute for Materials Science, 3-13 Sakura, Tsukuba 305-0003, Japan \\ ** Joule Physics Laboratory, IMR, University of Salford, Manchester M5 4WT, UK
}

Inert gases sometimes behave in strange manner. Xe, for instance, becomes solid nano-crystals when it is ion-implanted into $\mathrm{Al}$ [1]. It is known that the precipitates with the size less than $5 \mathrm{~nm}$ in $\mathrm{Al}$ have an FCC structure, aligned with the matrix. The lattice parameter of this solid Xe is 50 percent larger than that of Al [2]. However, these crystals sometimes melt to fluid under electron irradiation under the observation in the electron microscopes. We used high-resolution transmission electron microscopy (HRTEM) and high-angle annular dark field scanning transmission electron microscopy (HAADF-STEM) to image and model an atomic structure of Xe inclusions $[3,4]$ and to investigate the interface structures when it is solid or fluid.

Ion beam implantation of Xe was carried out into Al TEM specimens at room temperature to a dose of $3 \times 10^{20}$ ions $/ \mathrm{m}^{2}$. HRTEM and HAADF-STEM were done with a JEM-ARM1000 and a 200-keV high-vacuum aberration-corrected JEOL-2500SES STEM equipped with a Gatan Enfina EEL spectrometer. Fig. 1 shows two large fluid cavities containing fluid Xe under the electron irradiation in the JEM-ARM1000 microscope [3]. The cavities are seen in $<110>$ projection with $\mathrm{Al}\{111\}$ and $\{100\}$ planes. Fringes are visible in the Xe at all or most facets containing fluid Xe. The fringes result from variation of contrast as illustrated in Fig. 2(A) for a $\{111\}$ facet (marked with an arrow). To interpret this intensity variation, atomic configurations resulting from MD simulations of a slab of fluid Xe (modeled as a dense Lennard-Jones fluid) are shown in fig. 2(B). The inset figures are the simulated images from which the contrast variations are derived. In both experiment and simulations, three clear fringes are observed. The origin of the fringes in the simulated images is the layering of $\mathrm{Xe}$ atoms in the fluid at the interface. This layering can be clearly seen in fig. 2(C) where the number of Xe atoms in the MD calculations in thin slices parallel to the interface is plotted as a function of distance. The results provide conclusive evidence for layering at a fluid/solid interface in a simple fluid.

STEM observations in Fig. 3 revealed a diameter range of $7-10 \mathrm{~nm}$ where crystalline, semi-crystalline, and amorphous Xe particles coexist in this range. In order to check whether chemical composition can account for this observation, we analyzed the EEL spectra in and around the particles. A part from the Al and Xe EEL peaks, oxygen $K$-edge signals at $530 \mathrm{eV}$ were detected and chosen for analysis and the representative results are summarized in Fig. 4. They revealed homogeneous oxygen distribution around crystalline and semi-crystalline particles and oxygen accumulation around the amorphous ones. Since Al surface is generally covered by about $4 \mathrm{~nm}$ of natural oxide, ion implantation not only introduces Xe but also recoils surface oxygen into the sample. TRIM calculations predict that in a "good quality" crystalline Al matrix covered by 4-nm oxide, the 30-keV Xe ions will be implanted at about $21 \mathrm{~nm}$ and the oxygen will be recoiled into Al to a depth $1 \mathrm{~nm}$. Therefore, oxygen will not reach Xe particles, and thus their structure will be crystalline. Therefore, the oxygen in the EEL profiles of crystalline and semicrystalline particles probably originates from the surfaces of the Al foil.

References

[1] R. C. Birtcher and W. Jäger, Ultramicroscopy 22, 267 (1987)

[2] K. Furuya, N. Ishikawa and C.W. Allen, J. Microscopy 194, 152 (1999)

[3] S. E. Donnelly, R. C. Birtcher, C. W. Allen, I. Morrison, K. Furuya, M. Song, K. Mitsuishi and U. Dahmen, Scienec, 296, 507 (2002)

[4] K. Iakoubovskii, K. Mitsuishi and K. Furuya, Phys. Rev. B, 78, 064105 (2008) 


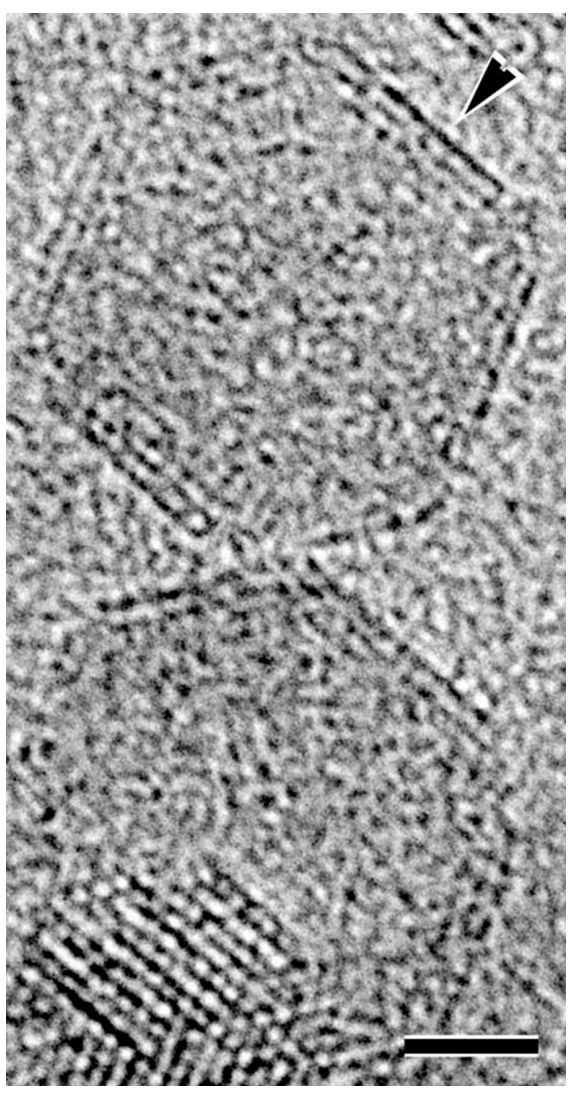

FIG. 1. HRTEM micrograph of facetted, Xe-containing cavities in $\mathrm{Al}$ at room temperature. The $\mathrm{Xe}$ in the two large cavities is fluid; in the small cavity at the bottom of the figure, $\mathrm{Xe}$ is solid. The scale marker indicates $2.5 \mathrm{~nm}$ [3].

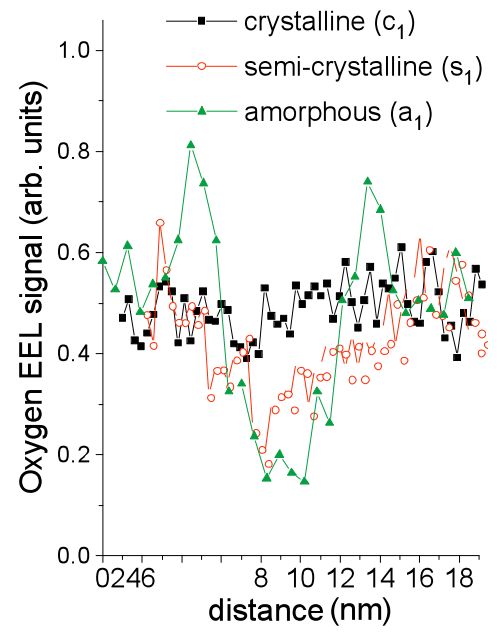

FIG.4. Representative line-scans of the integrated oxygen K-edge EEL signal acquired across a crystal. $\left(c_{1}\right)$, semi-crystalline $\left(s_{1}\right)$, and amorphous $\left(a_{1}\right)$ particles of Fig.3. Oxygen accumulation at the $\mathrm{Xe} / \mathrm{Al}$ interface is revealed for the amorphous particles [4].
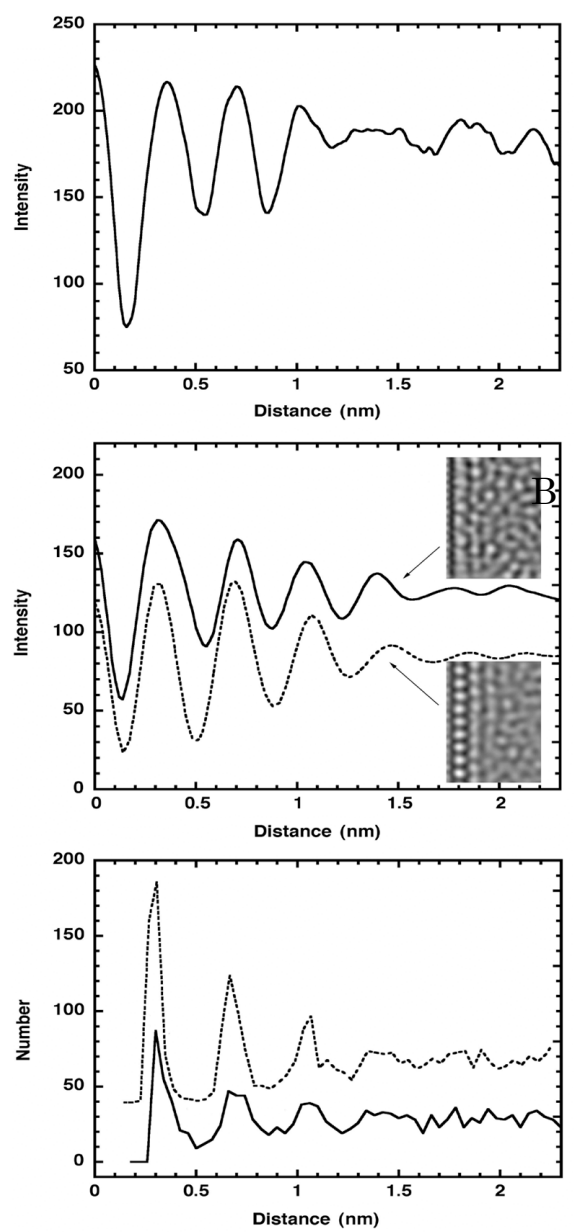

FIG. 2. (A) Variation in intensity as a function of distance within the cavity measured in a direction normal to the facet. (B) Intensity variation in two simulated HRTEM images of an interface between fluid Xe and solid Al. The inset images are the areas of the simulated images from which the intensity variation has been taken. (C) Number of Xe atom centres in $0.04 \mathrm{~nm}$ slices parallel to the $\mathrm{Xe} / \mathrm{Al}$ interface in the molecular dynamics simulations using "corrugated" (dashed curve) and "flat" (solid curve) potentials respectively [3]. 